\title{
Band Steering Technology Based on QoE- Oriented Optimization in Wireless Network
}

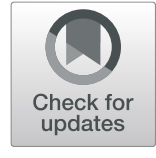

\author{
Zhifeng Han*, Jianxin Liao, Qi Qi, Haifeng Sun and Jingyu Wang
}

\begin{abstract}
In mobile crowd-sensing (MCS) network, it is necessary to reduce the cost of sensing data transformation from mobile devices to the MCS platform in the core of the network. With the widespread deployment of access point (AP), explosive data traffic of the mobile devices is offloaded from cellular base stations to Wi-Fi networks. The congestion of the $2.4 \mathrm{GHz}$ band in dual-band wireless area network makes the lower quality of experience (QoE) of users. To ease the load of $2.4 \mathrm{GHz}$ band, the band-steering technology has been widely used by equipment manufacturers. However, the fixed threshold configuration strategy in band steering technology makes it easy to be exploited by setting up a great number of connection requirements in a short time, which may prevent a normal connection requirement of a user, making it difficult to satisfy the dynamic resource requirements of different services. Moreover, if the threshold is configured too high, it may result in QoE imbalance in dual bands. Besides, when the band (such as $2.4 \mathrm{GHz}$ ) resources cannot meet the terminal requirement, the terminal cannot autonomously switch to another band (such as $5 \mathrm{GHz}$ ), and this may result in a very poor QoE of users. In this paper, a secure QoE-oriented wireless network optimization framework is proposed to improve the QoE of users in dual-band network. Firstly, to ensure the overall QoE of network and balancing the QoE between $2.4 \mathrm{GHz}$ and 5 $\mathrm{GHz}$ bands, the framework dynamically adjusts the load threshold of the frequency band based on QoE information during the association of a terminal. Secondly, to meet the basic QoE requirement of each user, the framework preferentially switches the terminals with poor QoE to the idle band after users join the network. The experimental results show that the proposed framework can improve the QoE both before and after the terminal accesses the network. Additionally, it enables the better QoE balance in dual bands.
\end{abstract}

Keywords: Mobile crowd sensing, Dual-band, Quality of experience, Wireless network, Access point

\section{Introduction}

\subsection{Background}

Mobile crowd sensing (MCS) is a kind of data collection approach which leverages the recent surge of sensorequipped smart mobile devices (e.g., smart phones, wearable devices, and tablets) to collect data. As the increasement of the sensing data, it is necessary to reduce the cost of sensing data transformation from mobile devices to the MCS platform in the core of the network. With the widespread deployment of access point (AP), explosive mobile application data traffic is offloaded from cellular base stations to Wi-Fi networks [1]. Quality of Experience (QoE) is defined as the overall acceptability of an application or service perceived

\footnotetext{
* Correspondence: Zhifeng.han@gmail.com

Institute of Network Technology, Beijing University of Posts and Communications, Beijing 100876, China
}

subjectively by the end users [2]. The increasing demand on wireless data communication requires effective approach that utilizes limited resource to achieve higher QoE. The necessity of selecting an optimal access point is likely to become once again a striking issue [3]. The method for Wi-Fi access point selection for most mobile devices is simple: most of the devices tend to leverage RSSI to connect to the Wi-Fi network with the strongest signal strength [4]. As a result, a client device that is capable of working on both frequency bands will typically prefer to connect to the APs on the $2.4 \mathrm{GHz}$ band [5]. However, when too many dual-band client devices connected to the $2.4 \mathrm{GHz}$ band, the efficiency of communication between the wireless access point and the client devices is diminished, which leads to the channel being congested and the QoE decreased [6]. 
In order to release the load of the $2.4 \mathrm{GHz}$ band, a technique called band steering [7] is widely used by several network service providers. By steering some client devices to the less frequently used $5 \mathrm{GHz}$ frequency band, data communication over the wireless portion of the network may become more efficient [8]. However, there are some shortcomings in band steering: Firstly, the band-steering technology determines whether a new user is allowed to join the network by comparing the band load with a fixed load threshold. While, in a high-rate data service environment, even the band load does not exceed the fixed threshold, the overall QoE of users may be poor, because high-rate data services like video and large file downloading consume more network resources [9]. Conversely, when the low-rate service like Web browsing occupies a major proportion in the network [10], even if the band load exceeds the threshold, the overall QoE of the network maybe maintained in a good state [11]. Secondly, bandsteering technology can only connect a dual-band user to the $5 \mathrm{GHz}$ band in the process of AP selection stage, if a user has already joined the network, it does not work. As a result, when the band resources cannot meet users' resources request, users' QoE will be decreased, and the band steering can do nothing in this condition. Thirdly, band-steering technology does not consider the traffic features of different applications. According to [2], there are four different QoS classes: the conversational class, the streaming class, the interactive class, and the background class [12]. The main distinguishing factor between them is how delay sensitive the traffic is.

Compared to the $2.4 \mathrm{GHz}$ band, the $5 \mathrm{GHz}$ band has a faster data transmission speed, higher channel capacity, which is more suitable to deal with the delay sensitive applications, such as real time audio/video, e.g., it is necessary to analyze the feature of applications, and then set up different steering policies for different applications to make different devices connect to $2.4 \mathrm{GHz}$ band or $5 \mathrm{GHz}$ band, respectively, which is the main motivation of our work.

\subsection{Related work}

Much effort has been dedicated to qualifying subjective user experience and constructing analytical models based on different experiment setup, such as [13, 14]. In [15], $\mathrm{SDN}$ is applied to the heterogeneous resource allocation in a multi-user scenario. However, a user can only connect to the closest Wi-Fi AP, excluding the possible associations with other Wi-Fi APs for better load balancing or higher overall QoE. Meanwhile, [16] and [17] focused on the optimization of QoE. Navarro-Ortiz et al. [16] proposed a QoE-aware scheduling scheme for the next-generation mobile network to minimize the total rebuffering time for all the users. In [17], a QoE-based cross-layer optimization framework for video delivery in LTE mobile networks is proposed. In order to deal with the problem that the total utility or the QoE drops when users compete for a single Wi-Fi AP, an application layer scheme [18] is proposed to adjust the target users and resources adaptively based on estimated users' states, and then the total QoE attained by all users is maximized.

There are also some researchers concentrated on the QoE of video streaming [19]. In [20], an objective metric to assess the QoE and obtain the relationship between this metric and the achieved MAC layer throughput is proposed, and this can make the MAC layer be aware of the QoE performance directly [21] proposed an online scheduling policy to optimize QoE for video-on-demand applications in wireless networks. It considers not only the long-term average performance but also short-term QoE performance.

The IEEE $802.11 \mathrm{k}$ amendment [22] also attempts to solve the load balancing problem, but requires extra modifications to the AP and the wireless client, which is not practicable for the commodity hardware. Authors in [23] present an efficient solution to control the user-AP associations for max-min fair bandwidth allocation which greatly reduce the load imbalance and consequent unfair bandwidth allocation. The proposed algorithms compute a fractional association solution, in which users can be associated with multiple APs simultaneously. This solution guarantees the fairest bandwidth allocation in terms of max-min fairness.

In [24], the author stated the reasons why there are few numbers of research that worked on AP connection management: (1) IEEE 802.11 standard is originally designed for the wireless extension of local area networks, and the public area with severe interference is not properly designed to handle user's needs; (2) Wi-Fi APs basically provide very limited types of feedback to mobile stations. In recent years, in order to ease the user load of 2.4 $\mathrm{GHz}$ band, a technique called band steering [7] is widely used by several major network service providers. The band steering has two major functions: one is spectrum navigation, and the other is load balancing. Through band steering, the dual-band clients are preferentially connected to the $5 \mathrm{GHz}$ band, and the number of users between $2.4 \mathrm{GHz}$ and $5 \mathrm{GHz}$ bands is relatively balanced. To provide acceptable Internet service for device users, an efficient mechanism of auto-connecting a mobile device to the best Wi-Fi AP [25], and it can guarantee the connected AP is much more qualified in serving the Internet services than an AP that is determined by the current connection. In [26], the authors posited that the recent introduction of frame aggregation by 802.11e can offer a compact and efficient representation of expected throughput for improving AP selection. The authors showed that by conveying the characteristics of subframes during frame aggregation, they can uniquely embody the utilization, interference, and backlog traffic 
pressure for an access point. Without modification on $\mathrm{AP}$, the NU methods take advantage of broadcasting nature of Wi-Fi to capture information by monitoring from client side. For example, SmartAssoc [27] required clients to hear the nearby packet transmission rate information. With the same principle, all algorithm designs rely on their AP measurement metric. Without a robust metric as the foundation, upper-level designs may mislead AP selection. Vasudevan et al. [28] proposed a metric that can be used for AP selection mechanism, and the metric is based on the expected bandwidth that can be achieved by each AP with a certain beacon reception delay. The paper argues that the arrival of a beacon from each AP is differed by the queuing delay of each beacon to be sent, and the queuing delay increases as the amount of data the AP should handle increases. Other than focusing on the quality of Wi-Fi based on link state estimation, Google Inc. [29] suggested an intelligent Wi-Fi selection engine that is based on past Wi-Fi information stored in database servers. For instance, if a user wants to use Wi-Fi for Internet service on his/her smartphone, the user phone requests the quality information of surrounding Wi-Fi APs to one of database servers located at infrastructure network. Quality information is given by prior $\mathrm{Wi}-\mathrm{Fi}$ users that provide transmission speed and delay information to servers.

\subsection{Methods and contributions}

In this paper, to improve the QoE of users in different mobile crowd-sensing service scenarios, we propose a novel QoE-oriented wireless network optimization framework. Our main contributions in this paper are summarized as follows:

- In order to monitor the user QoE in the network dynamically, we design a QoE-oriented optimization framework, which consists of three modules: QoE sensing, flow monitoring, and strategy scheduling. The framework adjusts the load threshold of a band based on overall QoE in different service scenarios and controls the terminals to switch from 2.4 to 5 $\mathrm{G}$, or vice versa.

- In order to quantify the QoE, we propose a QoE assessment model for multi-service scenarios, which consists of indicator layer, criterion layer, and target layer [30]. Further, the scorning mechanism is presented to implement non-dimensional treatment of key quality of indicator (KQI).

- To prove the availability of our QoE-oriented optimization framework, we provide the experiment to compare the performance of our framework. The overall QoE of network and QoE distribution of individual terminal are considered in this comparative experiment. The experimental result shows that the proposed framework can better improve the QoE in different service scenarios and can enable the QoE balance in dual bands.

The rest of this paper is organized as follows. Section 2 introduces the related work. Section 3 formulates the problems and gives the necessary definitions. Section 4 describes our QoE-oriented optimization framework in details and introduces the calculation model of QoE. Then, the experiment is provided to prove the availability of proposed framework in Section 5. Finally, the conclusions are drawn in Section 6.

\section{Design goals and design issues}

We will discuss our design goals and design issues in this section.

\subsection{Design goals}

\subsubsection{QoE sensitive}

The system has to be sensitive to users' QoE fluctuation, and it should have a quantized method to evaluate the QoE. It should collect the QoE-related data and extract the correspondent results in real time, and the system also needs to make the QoE optimization decision in real time based on the QoE status of a user.

\subsubsection{Dynamic connection control}

The control strategy of band steering should be dynamical. It should not only control the connection of new coming devices but also monitor and steer the already-in devices. The system should keep users in $2.4 \mathrm{GHz}$ band and $5 \mathrm{GHz}$ band experience all in a nearly same level. Concerned to an already-in device, if it has a poor QoE, the system should change it to another band in real time.

\subsection{Design issues}

Based on the design goals discussed above, there are following design issues to be solved.

- How to quantify the QoE? As it is known to all, QoE is the quality of experience when a user experiencing a service, which is subjective and can hardly be described directly. For this reason, there are different QoE evaluation models constructed. Moreover, different parameters need to be collected for different services. Therefore, to evaluate the QoE, it is necessary to understand what kind of QoE a user is interested in and what kind of parameters related to the correspondent service that are needed to be collected.

- How to monitor the QoE- or the QoE-related data in the system? In order to improve the QoE, it is necessary to collect the relevant parameters related to the QoE in real time. In the proposed architecture, 
it needs to consider what kind of data needs to be collected, how to deploy the monitoring module in the architecture and collect these data in real time, how to change the raw data into the specific parameters.

- How to improve the QoE under circumstances? As we have already the QoE evaluation model and also collected the related parameters, the last problem is how to leverage the related information to make the control decisions in reactive. How to design our architecture and how to implement the control model in it.

\section{Proposed framework}

\subsection{System framework overview}

Figure 1 shows the proposed QoE-oriented system framework. As shown in Fig. 1, it consists of three modules, which are QoE-sensing module, flow monitoring module, and the strategy scheduling module.
The QoE-sensing module is responsible for collecting the original data. Compared to the traditional data collecting method, this architecture leverages the mobile terminals to collect QoE-related data, which provides a reliable data basis for QoE evaluation. The flow monitor module recognizes the traffic flow of various services and records them in log server, then all data is uploaded to the strategy-scheduling module eventually. The strategyscheduling module consists of data analysis platform, the Agent for QoE control, and strategy template library [31]. The data analysis platform is responsible for statistical analysis of log data, computing the QoE-related information of the entire network. The Agent plays a core part in our QoE-oriented optimization framework. It consists of data interface, service interface, and control interface. The data interface receives the data stream to ensure that the Agent can master the global network state in dual-band environment. Then, the data stream of multi-service will be further analyzed and compared in service interface. The result is helpful for the Agent to quickly confirm the

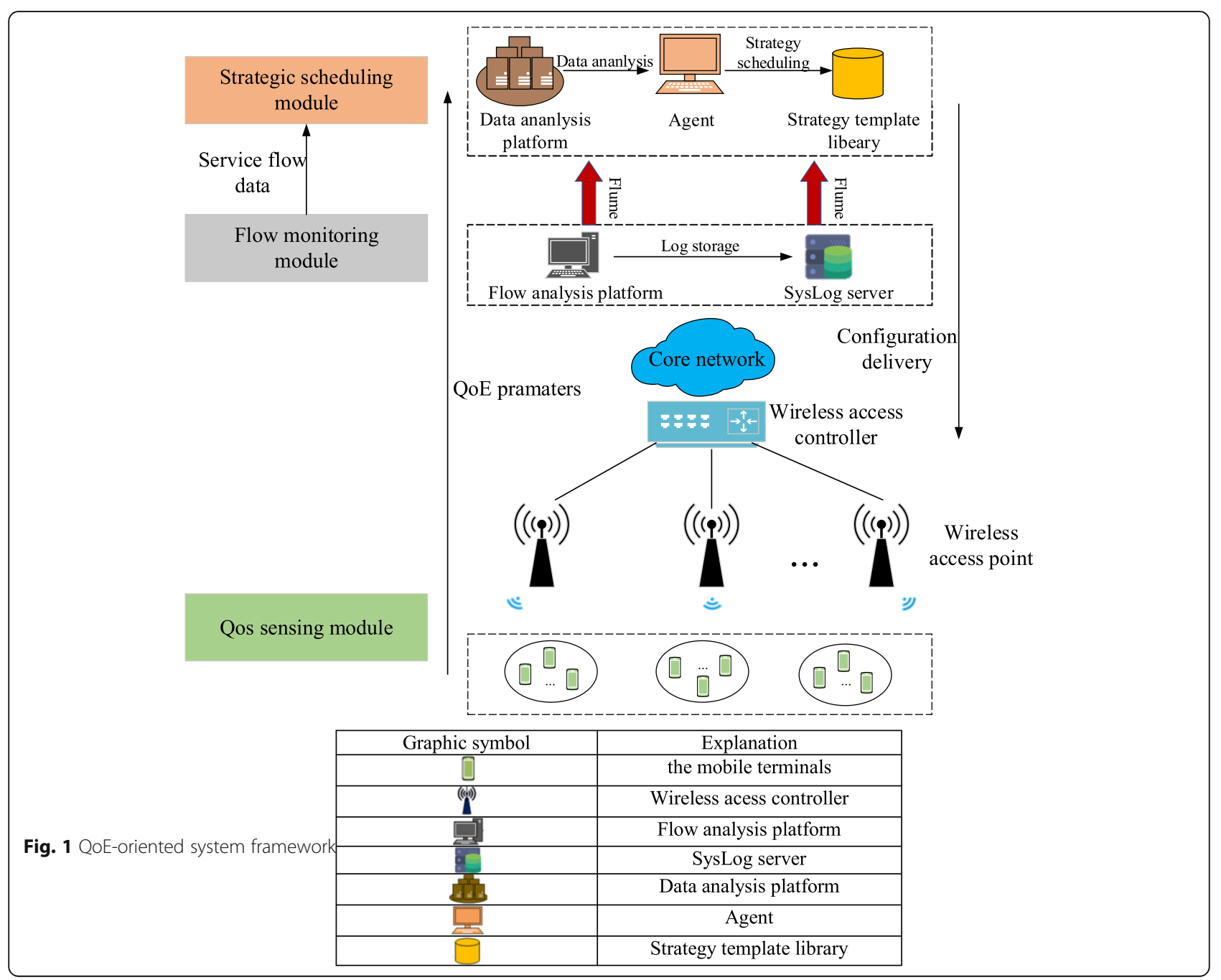


service scenarios and accurately match the optimization strategies that satisfies the service requirements [32]. The control interface of the Agent delivers a policy-mapping table to the wireless access controller (AC) so as it can schedule the strategies in template library. Thus, the strategies for load threshold adjustment and band switching can be scheduled to implement adaptive optimization of the wireless network [33].

\subsection{System functional architecture}

In order to better illustrate the main functions of our architecture, we divide the architecture into small function modules, which is described in Fig. 2.

\subsubsection{The QoE-sensing module}

In our architecture, the proposed framework formulates the optimization strategies based on QoE, and the collection of QoE-related parameters becomes the most important work. As the direct carriers of data services in wireless network, mobile terminals can be used to capture service data from user side [34], which can reflect the quality of each specific application more accurately. However, this method may involve the actual user behavior, leaking parts of privacy and data security of users to some extent [35-38]. Therefore, in our framework, the QoE-sensing module extracts KQI data by simulating current mainstream services like webpage browsing, video watching, and file downloading. The module can further divide its functions into logic service processing part and data service processing part according to a service processing type. In the logic service processing part, a simulating mobile terminal will first collect central frequency information of Wi-Fi signals and then decide whether it will connect to the $2.4 \mathrm{GHz}$ frequency band or the $5 \mathrm{GHz}$ band. Later, the terminal will finish different simulations under different types of network services in this frequency band. In data service part, the sensing module will collect the KQI data during the simulation in real time and then processes and calculates the KQI parameters in the backstage. Finally, the QoE evaluation results are uploaded to the strategy scheduling module for further analysis.

\subsubsection{Flow monitoring module}

The flow monitoring module is responsible to identify and classify the flow of multi-services in mobile terminals of in the network [39], which can map the service throughput into QoE in real time. The original methods detecting and classifying the service flow are always according to the network layer metrics, such as IP address and communication ports. This is because the fixed well-known ports, especially below 1024, are widely used by many traditional application protocols. For example, the HTTP protocol uses the port 80 for communication. Therefore, the traffic distribution which uses different protocols in application layer can be analyzed through the communication ports below 1024. However, to avoid being detected by the network access control policy

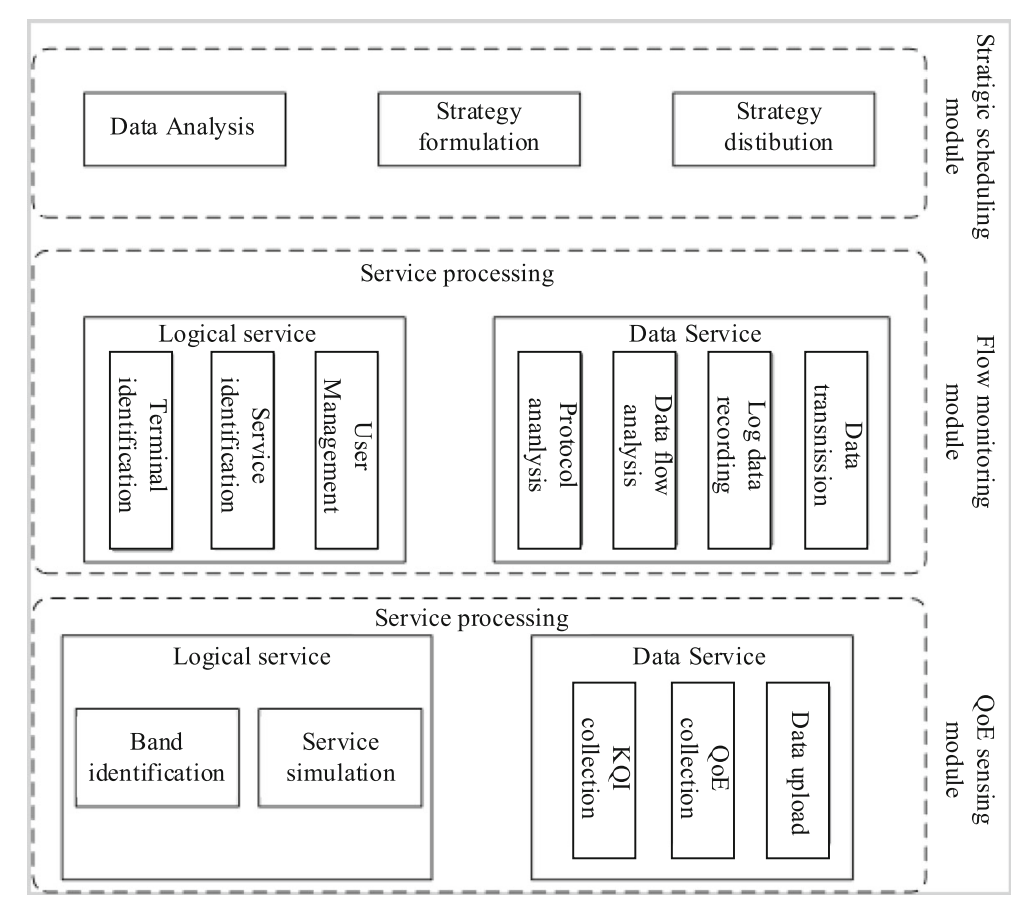

Fig. 2 Functional framework of the system 
enforced by the firewalls, a large number of application software no longer use fixed ports. For example, the Skype uses the ports 80 and 443 for communication so that the flow detected through port 80 may not all come from the HTTP protocol. Therefore, the flow detection method based on the network layer metrics cannot be fully applied to current Internet technologies.

In our proposed framework, the framework uses DPI technology and Deep Flow Inspection (DFI) [40] to accurately identify service flow in wireless LANs. DPI is a flow detection method in application layer, and it can recognize various protocols in application layer through the content detection of the network packet. Moreover, it can capture the raw data packet based on application layer metrics such as feature values, application protocols, and behavior patterns. However, DPI cannot identify encrypted application protocols. To solve this problem, we leverage DFI which is based on flow behavior to identify applications in networks, which identifies a session-based application through behavioral characteristics and is suitable for detecting encrypted application protocols. Different applications have different data flow status. For instance, the IP voice flow has features that the packet length ranges between 130 and 220 bytes, and the flow rate reaches $20 \sim 84 \mathrm{kbit} / \mathrm{s}$. However, the flow behavior of P2P-based application includes four aspects: the average packet length is greater than 450 bytes, the download duration is long, the link speed is fast, and the preferred transport protocol is TCP. In our framework, we use the DFI to construct the feature model based on the flow behavior and to identify the application types [41], the behavior of session flow is analyzed, which includes the packet length, link speed, the amounts of transmitted bytes, and the packet interval. Then, the analysis results are compared with the feature model [42]. In summary, the DPI and DFI work together in this module can identify different service flow, and the identified flow data are recorded in the SysLog Server and transmitted to the strategic scheduling module for further analysis.

\subsubsection{Strategy-scheduling module}

The strategy-scheduling module is the core function in our proposed architecture. It is mainly responsible for analyzing the flow data, scheduling optimal strategies, and distributing optimal strategies to AC. Initially, the data analyzing platform deployed on the strategyscheduling module extracts different the throughout data of different applications from the network and individual terminals and then send the analyzed results to the Agent [43]. The Agent masters the global QoE information according to the mapping relationship between the throughput and QoE test results in mobile terminals, and then, the optimization strategies from the template library formulated before is selected [44]. To ensure the overall QoE being maintained above moderate level in different service scenarios, if the overall QoE of the network is lower than the MOS mid-level, the load threshold of the frequency band will be decreased; otherwise, the load threshold of the frequency band will be increased, and in this way, the overall network QoE is guaranteed, as well as allowing the number of connected users is as many as possible. Furthermore, for some terminals which have already connected in a certain band, the Agent will sort their priority for band switching based on their own QoE, and the mobile terminals with poor QoE value will be preferentially switched to another band. To accomplish dynamic load threshold adjustment and frequency band switching of the terminal in the dual-band network environment, all the optimization strategies are sent to the wireless controller automatically through the Hyper Terminal in the Agent.

\subsection{The QoE evaluation model based on AHP}

\subsubsection{The construction of QoE evaluation model}

We proposed a QoE evaluation model based on analytic hierarchy process (AHP) [45] for multi-service scenario. As shown in Fig. 3, our three-layer model consists of the target layer, the criterion layer, and the indicator layer. The target layer represents the overall QoE of all related services in the network. In the criterion, each element represents the QoE of a specific service, and the children of which the indicator layer are the key quality indicators (KQIs) related to its service [46].

In our proposed QoE evaluation model, the overall QoE in the target layer is the weighted sum of different sub-QoEs in the criterion layer [47], that is:

$$
\mathrm{Qo} E=\omega_{1} * \mathrm{Qo} E_{1}+\omega_{2} * Q \mathrm{o} E_{2}+\cdots+\omega_{n} * Q \mathrm{o} E_{n}
$$

where $\omega_{i}$ is the weight of a sub-QoE. The $Q o E_{i}$ corresponding to a service is calculated as follows:

$$
Q o E_{i}=\omega_{1}{ }^{\prime} * K Q I_{1}+\omega_{2}{ }^{\prime} * K Q I_{2}+\cdots+\omega_{n}{ }^{\prime} * K Q I_{n}
$$

where $\omega_{i}^{\prime}$ is the weight of the KQI.

The weights of the sub-QoE in Eq. (1) and the weights of the KQIs in Eq. (2) can be calculated by constructing an AHP matrix [46]:

$$
A=\left[\begin{array}{cccc}
a_{11} & a_{12} & \cdots & a_{1 j} \\
a_{21} & a_{22} & \cdots & a_{2 j} \\
\vdots & \vdots & \ddots & \vdots \\
a_{i 1} & a_{i 2} & \cdots & a_{i j}
\end{array}\right]
$$

where $a_{i j}$ represents the importance of each element pair. By calculating the maximum eigenvalue $\lambda_{\max }$, the normalized eigenvector of $\lambda_{\max }$ corresponds the weights of the elements (the sub-QoEs or the KQIs) constructing the overall QoE of the sub-QoE. 


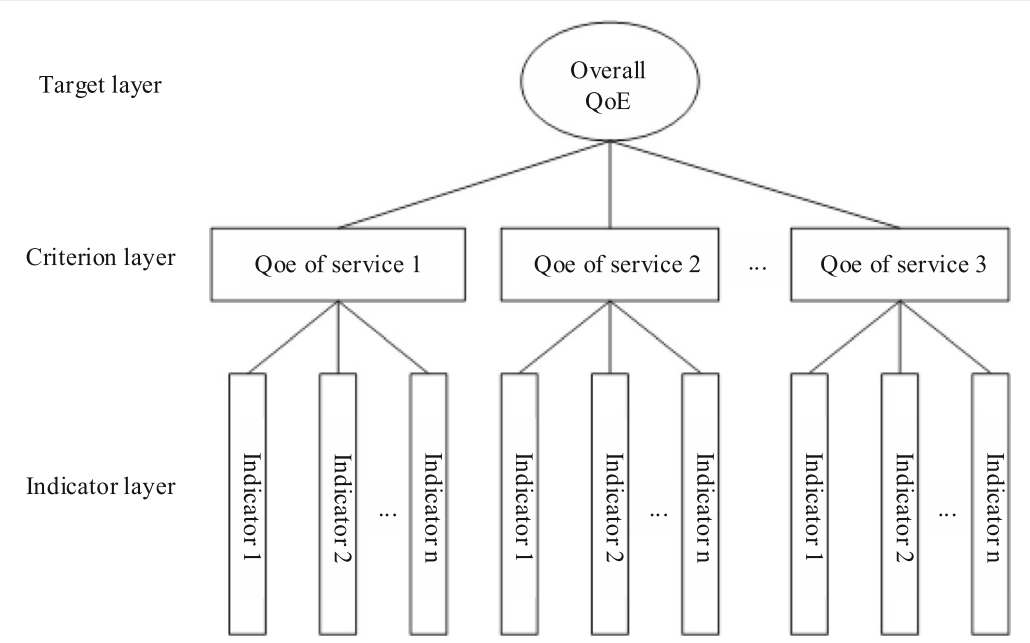

Fig. 3 QoE assessment model for multi-service scenarios

\subsubsection{The KQI scoring mechanism}

The KQIs cannot be compared directly with each other, as each has its own physical meanings. For instance, although the units of both DNS resolution delay and the first packet delay are millisecond, they express different physical meanings. Therefore, it is necessary to implement non-dimensional treatment of KQIs for different services. The common non-dimensional method is to calculate score of each KQI $H\left(x_{m}\right)$ through the standardized processing of measurement value. In this paper, the measured indicators were normalized by the method of maximum and minimum values.

\subsubsection{Determination of the evaluation thresholds} Normalized results of KQI can be mapped between 0 and $N$, where $N$ is the full score of KQI and corresponds to the value 5 in the MOS evaluation method. As shown in Fig. 4, the $T h_{\max }$ denotes the upper limit value of the measured indicators and the $T h_{\min }$ is the lower limit value. To determine the measurement threshold, the measurement data of a KQI are arranged in the ascending linear order (same numerical values are eliminated) and then divided into five parts according to MOS level. The first part is corresponding to the lower limit value and the fourth part is corresponding to the upper limit value.

\subsubsection{Mapping from measured values to KQI scores}

The KQIs can be further divided into the positive indicators and negative indicators. The former one refers to KQIs that, the higher of its value, the better meaning it represents, such as download success rate. The later one refers to KQIs that, the higher of its value, the worse meaning it represents, such as delay time.

The mapping relationship for positive indicators is that, if KQI $T$ is higher than the $T h_{\max }$, it will be scored with a value $N$, and if KQI is lower than the $T h_{\text {min }}$, it will be scored with a value 0 . Moreover, when the numerical value of KQI ranges from $T h_{\min }$ to $T h_{\max }$, it will be mapped into the interval of $[0, N]$ according to the linear ratio, and the mapping function can be expressed as:

$$
H\left(x_{m}\right)=N-\frac{N}{T h_{\max }-T h_{\min }} \times\left(T h_{\max }-T\right)
$$

The mapping relationship for negative indicators is that if KQI $T$ is higher than the upper limit value, it will be scored with a value 0 , and if the KQI does not reach the lower limit value, it will be scored with a value $N$. When the numerical value of KQI ranges from $T h_{\text {min }}$ to $T h_{\max }$, it will be mapped into the interval of $[0, N]$ according to the linear ratio, and the mapping function can be expressed as:

$$
H\left(x_{m}\right)=N-\frac{N}{T h_{\max }-T h_{\min }} \times\left(T-T h_{\min }\right)
$$

3.3.2.3 Deal with the extreme deterioration of indicator According to (4) and (5), when the measured value of KQI is far more than the range of the threshold, the result of $H\left(x_{m}\right)$ will be an approximate constant.

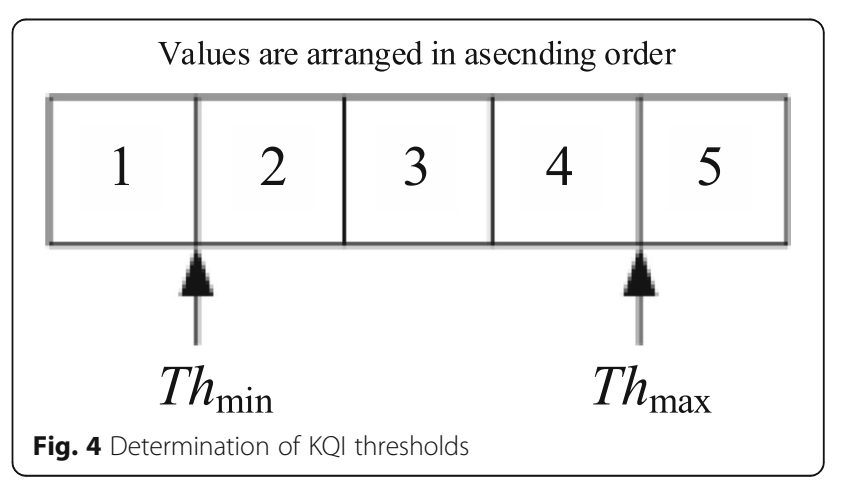


Actually, the extremely deteriorated indicators will have a great impact on the scoring result. To avoid such problem, the $H\left(x_{m}\right)$ has to be corrected.

For positive indicators, the corrected results are expressed as:

$$
\begin{aligned}
& -k, T h_{\min } \geq k T, k=1,2,3 \cdots, N \\
& \begin{aligned}
H\left(x_{m}\right)=\{ & N-\frac{N}{T h_{\max }-T h_{\min }} \times\left(T h_{\max }-T\right), T h_{\min } \leq T \leq T h_{\max } \\
& N, T>T h_{\max }
\end{aligned}
\end{aligned}
$$

where the result of $H\left(x_{m}\right)$ is $-k$, when the measured value $T$ is far lower than $T h_{\text {min }}$.

For negative indicators, the corrected results are expressed as:

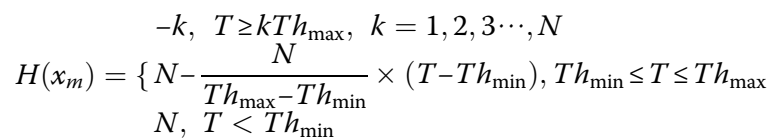

where the result of $H\left(x_{m}\right)$ is $-k$, when the measured value $T$ is far higher than $T h_{\max }$.
Then, the standardized value of KQI can be used to calculate the QoE directly, thus getting the final assessment result.

\section{Experiment and result analysis}

In this section, we implemented the proposed SDN-based AP selection mechanism in both an Android phone and an experimental wireless network. In order to illustrate how effective of our proposed method, we compare different QoE parameters under the same experiment environment, and the compared approaches are the original state approach and the band-steering approach. The original state approach means a device accesses an AP directly, and the AP has no corresponding optimal ways to steering the devices according the access load. The band-steering approach means that the AP will adjust devices from 2.4 to $5 \mathrm{G}$, or from 5 to $2.4 \mathrm{G}$ according the traditional banding steering policy embedded in the AP. Then, we carried out a QoE performance evaluation study to verify the effectiveness of the proposed scheme.

\subsection{The experimental wireless network}

The experimental network topology is shown in Fig. 5. A smart application gateway named Panabit [48] is deployed

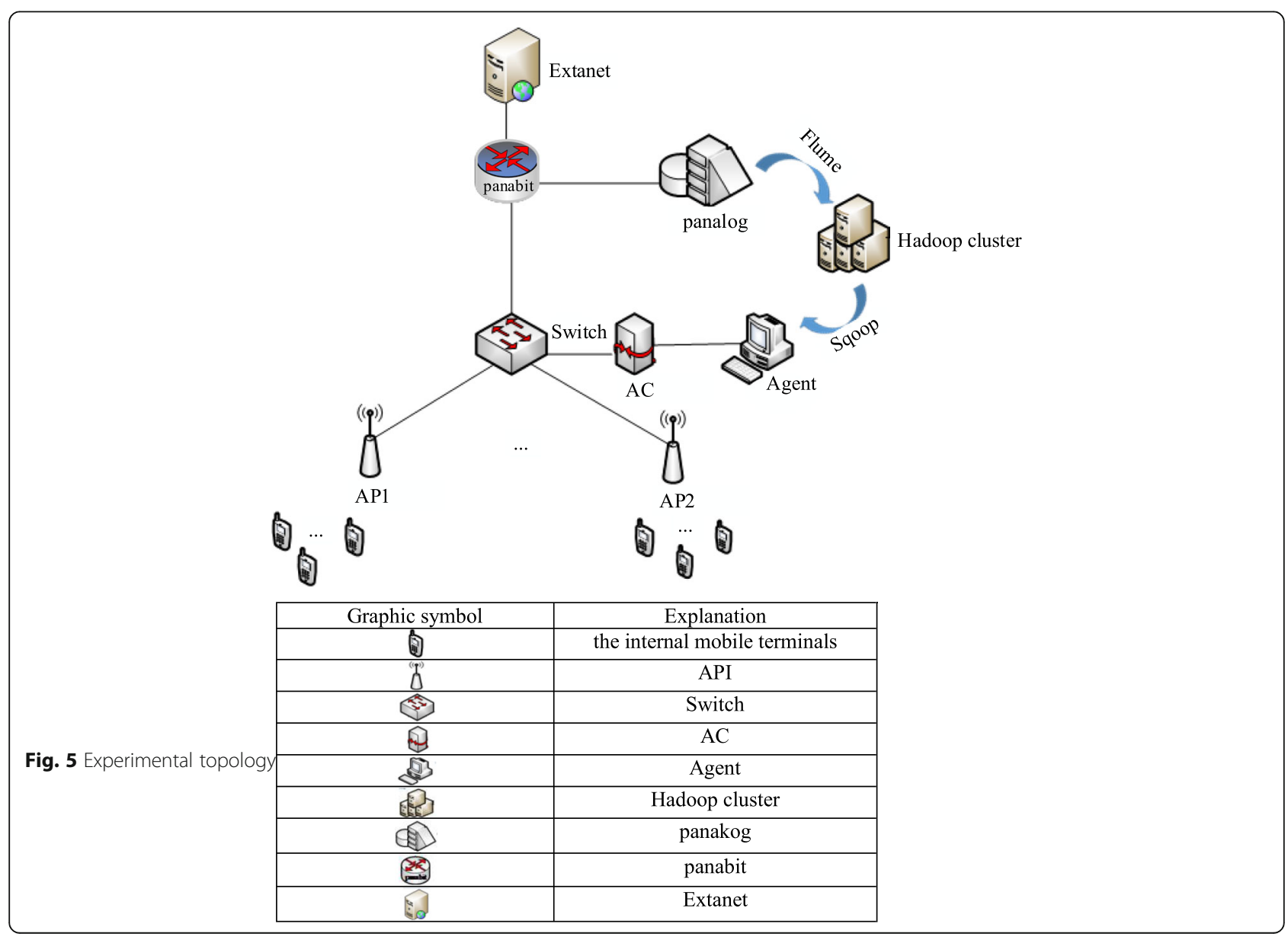


Table 1 Experimental setup

\begin{tabular}{ll}
\hline Parameters & Parameter settings \\
\hline The number of dual-band AP & 1 \\
Total bandwidth of AP & $4 \mathrm{M}$ \\
The number of smart phones & 5 \\
Data service type & High/low-speed service \\
\hline
\end{tabular}

between internal and external networks. The Panabit with routing mode plays two roles in our experiment. On the one hand, the routing strategy is formulated on Panabit to map the internal IP address into external network, which can ensure the internal mobile terminals access to different services successfully. On the other hand, the Panabit can identify different kinds of application layer data packets and will also extract the IP address, protocol type, e.g., and the results are saved in a log storage system named Panalog [48]. The network adopts Flume [49] technology to transmit the log data to Hadoop cluster for further analysis [50]. Furthermore, the analyzed results of the service throughput are fed back to our Agent by the Sqoop [51] technology. The Agent triggers the automated scripts in the hyper terminal named Minicom [52] and schedules the steering strategies from the template library to $\mathrm{AC}$. In this way, the QoE of a wireless network optimization is realized.

\subsection{Experimental setting}

\subsubsection{Basic parameter setting}

The experimental parameters in this paper are listed in Table 1. It needs one AP which supports the dual-band network, and the bandwidth of AP was set to $4 \mathrm{M}$. In the experimental process, five smart phones are accessing to the frequency band network. The service scenarios include high-speed service and low-speed service, such as large file downloading, webpage browsing, and video viewing.

\subsubsection{QoE calculation in our experimental environment}

Due to the limitations of the experimental environment, we only consider three types of services such as webpage browsing, video, and download service. As shown in Fig. 6, we chose different KQI parameters to construct our QoE evaluation model. The KQI parameters for webpage browsing service include DNS resolution delay, first packet delay, and the delay to open the webpage. The KQI parameters for video service include the initial cache time, re-buffering frequency, and average buffering delay. The KQI parameters for download service include the average download rates, peak download rate, and download success rate.

Take the webpage browsing service as an example, and the judgment matrix can be constructed as follows:

$$
A_{1}=\left|\begin{array}{ccc}
1 & 1 / 3 & 1 / 4 \\
3 & 1 & 1 / 2 \\
4 & 2 & 1
\end{array}\right|
$$

Therefore, the maximum eigenvalue is $\lambda_{\max 1}=4.104$, and the corresponding normalized eigenvector is $\omega_{i}^{\prime}=$ $\left[\begin{array}{lll}0.122 & 0.320 & 0.558\end{array}\right]^{T}$. The consistency ratio of the judgment matrix is $c r^{\prime}=0.013<0.1$, which implies that the judgment matrix is reasonable. Then, the QoE for the webpage browsing service can be expressed as follows:

$$
\begin{aligned}
Q o E_{1}= & 0.122 * K Q I_{1}+0.320 * K Q I_{2} \\
& +0.558 * K Q I_{3}
\end{aligned}
$$

In this way, we can calculate the QoEs for the video service, the downloading service respectively, and the overall QoE.

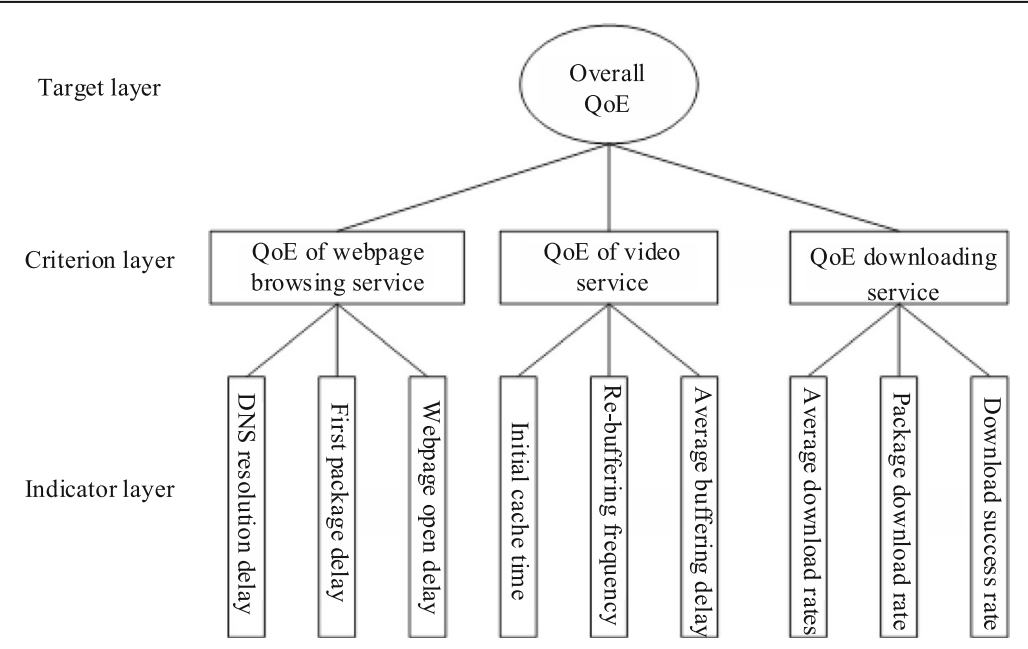

Fig. 6 QoE evaluation model in our experiment 


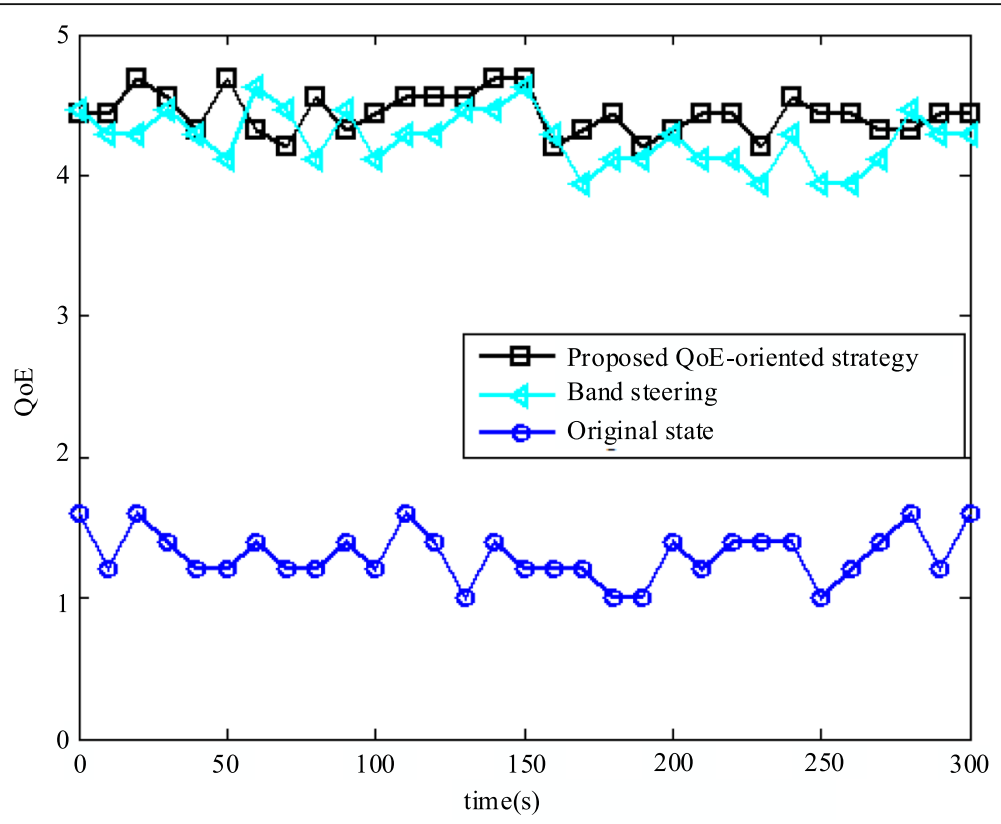

(a) Overall QoE in network with low-speed service

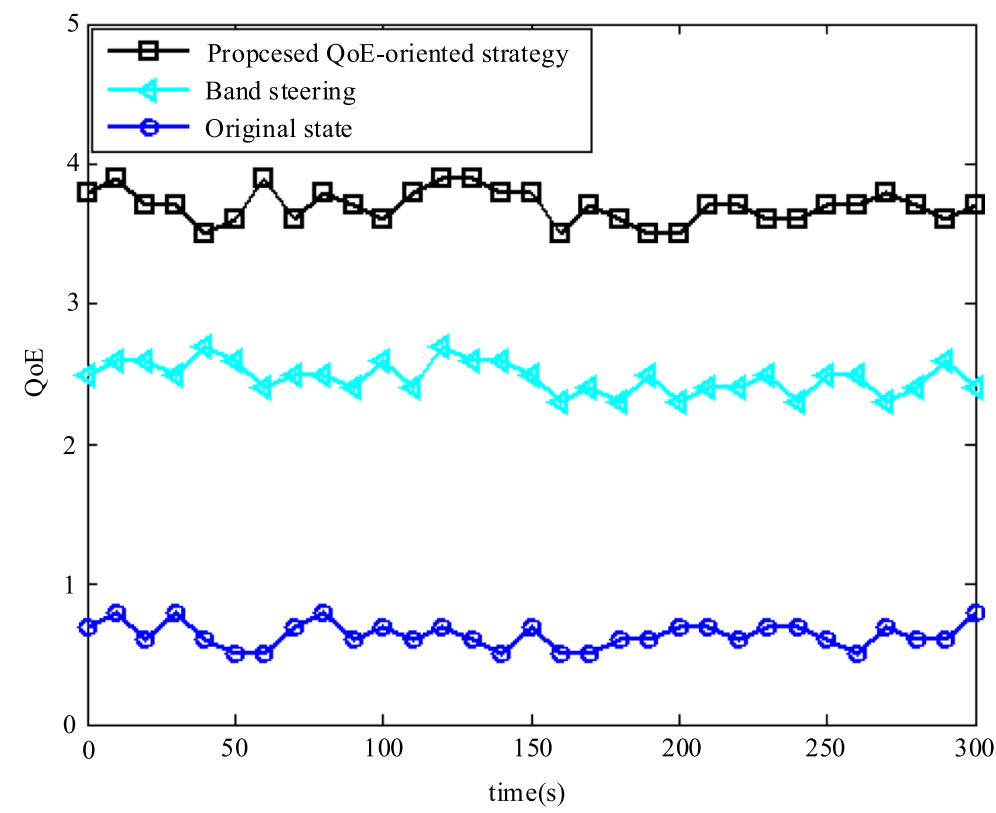

(b) Overall QoE in network with high-speed service

Fig. 7 Overall QoE of users in the network. a Overall QoE in network with low-speed service. b Overall QoE in network with high-speed service

\subsubsection{Evaluation parameters}

Definition 1: the overall QoE Q of users in the network: the overall QoE indicates the service satisfaction of all users in $2.4 \mathrm{GHz}$ and $5 \mathrm{GHz}$ bands. It can reflect the quality of service which offered by a dual-band AP.

Definition 2: QoE difference between band $2.4 \mathrm{GHz}$ and band $5 \mathrm{GHz}$ : The concept of QoE difference is proposed for QoE imbalance between $2.4 \mathrm{GHz}$ and 5 $\mathrm{GHz}$ band. It reflects that each frequency band provides different service experience to users.

Definition 3: QoE distribution: the QoE distribution is defined as the number of users that enjoy the moderate QoE. It can represent the ability of the network to meet basic QoE requirements. 


\subsection{Experiment results}

The main function of our framework for network optimization is to schedule the QoE-oriented strategies in different service scenarios. To verify the effectiveness of our framework, we have compared our QoE-oriented strategy with the situation that the AP without any selection strategy (the original state) and the AP open the band-steering function (the band steering). We observed the overall QoE of network and QoE distribution of the individual terminal under these three conditions. There are 5 smart phones associated with the dual-band AP, and they request different kind of services. The services include high speed data service and low speed data service. In our experiment, the former one refers to service like video or downloading, and the later one is webpage browsing service.

The results of overall QoE in our experiment are shown in Fig. 7. As shown in Fig. 7a, for the AP with original state, the overall value of QoE is stable at around 1.5, which means that the quality of user experience is poor. The reason is that all the devices will connect to the 2.4 $\mathrm{GHz}$ band with strong signal strength, but the limited bandwidth cannot meet the service requirements from the client devices. For the AP with band steering, the overall value of QoE is stable at around 4.2, and it means the quality of user experience is very good. For some client devices connected to $5 \mathrm{GHz}$ band, which mitigated from the congested $2.4 \mathrm{GHz}$ band, the load is balances in both $2.4 \mathrm{GHz}$ and $5 \mathrm{GHz}$. For AP with our QoE-oriented strategy, the overall value of QoEs is close to the result of band steering. This means that under the low-speed application services, the band steering and our QoE strategy tend to be nearly the same performance.

However, as shown in Fig. 7b, the overall value of QoE decreases when the application service changes into highspeed service scenario. As shown in Fig. 7b, the overall value of QoE with band steering drops to about 2.5, which means that the user experience deteriorated from very good to moderate level. The reason for this result is that the client devices requiring high-speed service scenario occupy too much network resources. However, the band steering allows a constant number of devices connect to the $5 \mathrm{GHz}$, and it cannot steer a device already connected to the 5 to 2.4 $\mathrm{GHz}$ band dynamically, even that device has a poor QoE in the $5 \mathrm{GHz}$. Compare to the band steering, the overall QoE value of the AP with QoE-oriented strategy decreases slightly under high-speed application service scenarios. The user experience is still good, which is because when a device performs a poor QoE, the agent will control the AP to steer the device to another band with a better network environment. In this way, it can improve the overall QoE of network in different service scenarios.

Additionally, in order to evaluate the QoE balance of our proposed framework, we compare the QoE difference in dual bands under three conditions, in which the association requests do not reach the load threshold. In this experiment, the QoE difference of a frequency band without any connected users will be defined as 5 scores, which means that the QoEs of users between two bands are much more imbalance. As shown in Fig. 8, with the increase of user load, the QoE difference in dual bands grows constantly under the condition that the network without any optimization strategies. Because all dual-band users are accessed to the $2.4 \mathrm{G}$ band, and the more user load, the worse QoE. Similarity, due to the priority feature of $5 \mathrm{GHz}$ band in band-steering technology, all dual-band users were accessed to the $5 \mathrm{GHz}$ band, the QoE difference between dual bands is significant. In comparison, our proposed framework switches the mobile user with pool QoE to idle band, when the QoE difference between dual bands exceeds one level in MOS model. The result proved the better QoE balance of our framework.

Besides, we also analyze the number of terminals whose QoE value is above the moderate level in low-speed data service and high-speed data service scenes, respectively, and the results are shown in Fig. 9. As illustrated in Fig. 9, the number of terminals with QoE value above the moderate level is recorded in the experiment. It decreases from 1 to 0 in the network that the AP without any strategy, and it decreases from 5 to 3 in wireless network that the AP with band steering. In comparison, all the terminal keeps a QoE value over the moderate level in wireless network that the with QoE-oriented steering strategy, because the strategy in our framework switches the terminals with poor QoE to another band dynamically. According to experimental results, the proposed framework can effectively control the QoE of individual terminal. However, due to the complexity of constructing an AHP matrix, the number of the weight parameter is not comprehensive, and the complexity of constructing a high dimension AHP matrix is much higher. In the future, we will focus on constructing a QoE evaluation model with more parameters and low complexity.

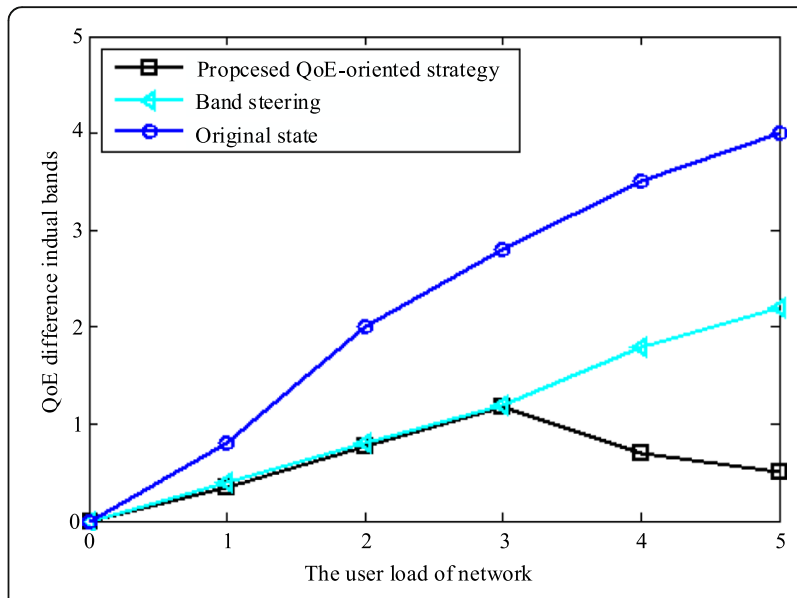

Fig. 8 QoE difference between $2.4 \mathrm{GHz}$ and $5 \mathrm{GHz}$ bands 


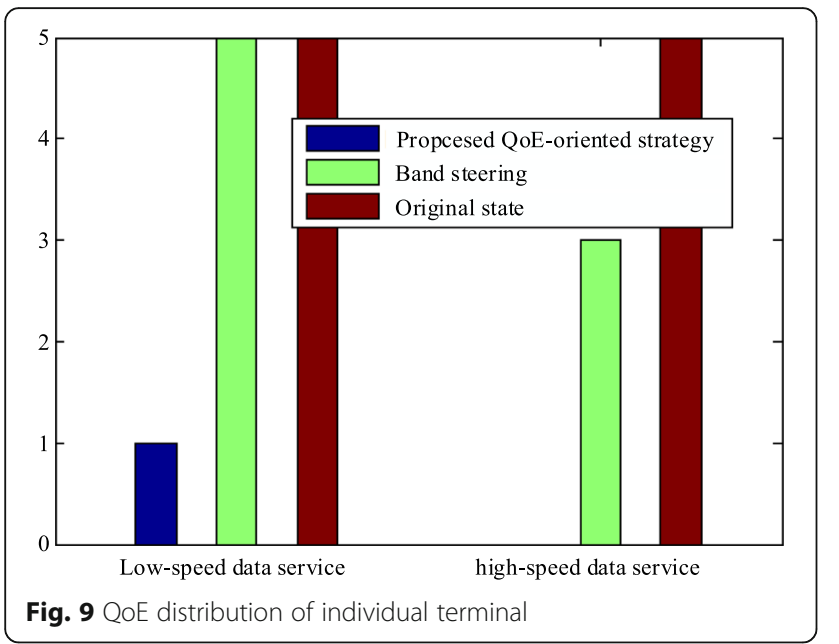

\section{Conclusion}

In this paper, a novel QoE-oriented framework for wireless network optimization is proposed, which aims to ease the QoE decrease due to the congestion of $2.4 \mathrm{GHz}$ band caused by the insecurity fixed threshold bandsteering configuration policy in dual-band wireless network environment. The framework is mainly composed of three modules: QoE sensing, flow monitoring, and strategy scheduling. Besides, in order to obtain the QoE result for multi-service scenarios, a three-layer QoE evaluation model based on AHP is proposed. Finally, the experiment is provided to prove the availability of our framework. The result shows that our framework is suitable for dynamic service scenarios in wireless network. It can improve the overall QoE of users in the wireless network and can enable that more terminal's QoE distributed above moderate level. Additionally, our framework could reduce the QoE imbalance between the $2.4 \mathrm{GHz}$ and $5 \mathrm{GHz}$ band.

\section{Abbreviations}

AC: Access controller; AHP: Analytic hierarchy process; AP: Access point; DFI: Deep flow inspection; DPI: Deep packet inspection; KQIs: Key quality indicators; QoE: Quality of experience; RSSI: Received signal strength indication

\section{Acknowledgements}

Not applicable.

\section{Authors' contributions}

ZH was a major contributor in writing the manuscript. All authors read and approved the final manuscript.

\section{Funding}

This work was jointly funded by (1) the National Natural Science Foundation of China (No. 61671079, 61771068, 61471063, 61421061, 61372120, 61271019;) and (2) the National Basic Research Program of China (No. 2013CB329102)

\section{Availability of data and materials}

The datasets generated and analyzed during the current study are not publicly available, but are available from the corresponding author on reasonable request.

\section{Competing interests}

The authors declare that they have no competing interests.

Received: 21 August 2019 Accepted: 2 January 2020

Published online: 28 January 2020

\section{References}

1. Q. Chen, G. Yu, H. Shan, A. Maaref, G.Y. Li, A. Huang, Cellular meets WiFi: traffic offloading or resource sharing? IEEE Transactions Wireless Communications 15(5), 3354-3367 (2016)

2. I.-T. SG12, Definition of quality of experience, COM12- LS $62-E$, TD 109rev2 (PLEN/12), Geneva, Switzerland, January, 2007.

3. Li, Shancang, and L. D. Xu. Chapter 6 - security in enabling technologies. Securing the Internet of Things:109-118 (2017).

4. L. Song, A. Striegel, Leveraging frame aggregation to improve access point selection (I 2017 IEEE Conference on Computer Communications Workshops, Atlanta, 2017)

5. H. Kim, W. Lee, M. Bae, H. Kim, Wi-Fi seeker: a link and load aware AP selection algorithm. IEEE Transactions Mobile Computing 16(8), 2366-2378 (2017)

6. L. Qi, W. Dou, W. Wang, G. Li, H. Yu, S. Wan, Dynamic mobile crowdsourcing selection for electricity load forecasting. IEEE ACCESS 6, 46926-46937 (2018)

7. Laroche, Stephane, Chi Thanh Hoang, and Gilbert Moineau, Band steering, U.S. Patent No. 8,655,278, 18 Feb, 2014.

8. Cisco, Bandsteering overview, https://documentation.meraki.com/MR/Radio_ Settings/Band_Steering_Overview. Accessed Feb 2018.

9. S. Ding, S. Qu, Y. Xi, A.K. Sangaiah, S. Wan, Image caption generation with high-level image features. Pattern Recognition Letters 123, $89-95$ (2019)

10. L. Qi, X. Zhang, W. Dou, C. Hu, C. Yang, J. Chen, A two-stage localitysensitive hashing based approach for privacy-preserving mobile service recommendation in cross-platform edge environment. Future Generation Computer Systems 88, 636-643 (2018)

11. K. Zhang, Non trivial solutions off fourth-order singular boundary value problems with sign-changing nonlinear terms. Topological Methods in Nonlinear Analysis 40, 53-70 (2012)

12. S. Li, L. Xu, H. Song, T. Chen, Privacy, data assurance, security solutions for Internet of Things (PASS4IoT): Guest editorial. IEEE IET Networks 7(5), 281-282 (2018)

13. N. Staelens, S. Moens, W. Van den Broeck, I. Marien, B. Vermeulen, P. Lambert, R. Van de Walle, P. Demeester, Assessing quality of experience of IPTV and video on demand services in real-life environments. IEEE Transactions on Broadcasting 56(4), 458-466 (2010)

14. K. Ricky, P. Mok, E.W.W. Chan, R.K.C. Chang, in Measuring the quality of experience of HTTP video streaming, ed. by 12th IFIP/IEEE International Symposium on Integrated Network Management (IM 2011) and Workshops. (Dublin, 2011)

15. S. Kang, W. Yoon, SDN-based resource allocation for heterogeneous LTE and WLAN multi-radio networks. J Supercomputing 72(4), 1342-1362 (2016)

16. J. Navarro-Ortiz, P. Ameigeiras, J. Lopez-soler, J. Lorca-Hernando, Q. PerezTarrero, R. Garcia-Perez, A QoE-aware scheduler for HTTP progressive video in OFDMA systems, Communications Letters. IEEE 17(4), 677-680 (2013)

17. S. Singh, O. Oyman, A. Papathanassiou, D. Chatterjee, J. Andrews, Video capacity and QoE enhancements over LTE, 2012 IEEE International Conference on Communications (ICC) (Ottawa, Canada, 2012)

18. W. Huang, D. Meng, J.-N. Hwang, J. Park, Y. Xu, W. Zhang, QoE based SDN heterogeneous LTE and WLAN multi-radio networks for multi-user access (IEEE Wireless Communications and Networking Conference (WCNC), Barcelona, 2018)

19. S. Ding, S. Qu, Y. Xi, S. Wan, A long video caption generation algorithm for big video data retrieval. Future Generation Computer Systems 93, 583-595 (2019)

20. Z. Hu, B. Li, M. Yang, Z. Yan, QoE-aware admission control and MAC layer parameter configuration algorithm in WLAN (2015 IEEE Wireless Communications and Networking Conference (WCNC), New Orleans, 2015)

21. P.C. Hsieh, I.H. Hou, Heavy-traffic analysis of QoE optimality for on-demand video streams over fading channels (The 35th Annual IEEE International Conference on Computer Communications, San Francisco, 2016)

22. IEEE, Wireless LAN medium access control (MAC) and physical layer (PHY) specifications amendment 1: radio resource measurement of wireless LANs, IEEE Std 802.11 k-2008 (Amendment to IEEE Std 802.11-2007) (2008), pp. 1-244

23. Y. Bejerano, S.J. Han, L. Li, Fairness and load balancing in wireless LANs using association control. IEEE/ACM Transactions on Networking 15(3), 560-573 (2007)

24. H. Kim, W. Lee, M. Baee, H. Kim, Wi-Fi seeker: a link and load aware AP selection algorithm. IEEE Transactions on Mobile Computing 16(8), 23662378 (2017) 
25. H.S. Kim, E. Kim, H. Kim, QoE-driven Wi-Fi selection mechanism for next generation smartphones (2012 The First IEEE Workshop on Enabling Technologies for Smartphone and Internet of Things (ETSIoT), Seoul, 2012)

26. L. Song, A. Striegel, Leveraging frame aggregation to improve access point selection (2017 IEEE Conference on Computer Communications Workshops (INFOCOM WKSHPS), Atlanta, 2017)

27. F. Xu, X. Zhu, C.C. Tan, Q. Li, G. Yan, J. Wu, SmartAssoc: decentralized access point selection algorithm to improve throughput. IEEE Transactions on Parallel and Distributed Systems 24(12), 2482-2491 (2013)

28. S. Vasudevan, K. Papagiannaki, C. Diot, J. Kurose, D. Towsley, Facilitating access point selection in IEEE 802.11 wireless networks (The 5th ACM SIGCOMM conference on Internet measurement, Berkeley, 2005)

29. M.D. Austin, K. Huber, S.K. Meredith, M.A. Salmon, R. Tipton, Wi-Fi intelligent selection engine, US Patent Appl. US8570993 B2 (2010)

30. L. Qi, X. Zhang, W. Dou, Q. Ni, A distributed locality-sensitive hashing based approach for cloud service recommendation from multi-source data. IEEE J Selected Areas Commun 35(11), 2616-2624 (2017)

31. X. Zhang, L. Liu, Y. Wu, The eigenvalue problem for a singular higher fractional differential equation involving fractional derivatives. Aplied Math. Comput 218(17), 8526-8536 (2012)

32. Z. Gao, D.Y. Wang, S.H. Wan, H. Zhang, Y.L. Wang, Cognitive-inspired classstatistic matching with triple-constrain for camera free 3D object retrieval. Future Generation Computer Systems 94, 641-653 (2019)

33. S. Li et al., Adaptive and secure load-balancing routing protocol for serviceoriented wireless sensor networks. IEEE Systems J 8(3), 858-867 (2014)

34. Z. Gao, H.Z. Xuan, H. Zhang, S. Wan, K.K.R. Choo, Adaptive fusion and category-level dictionary learning model for multi-view human action recognition. IEEE Internet Things J (2019). https://doi.org/10.1109/JIOT.2019. 2911669

35. S. Li et al., Risk assessment for mobile systems through a multilayered hierarchical Bayesian network. IEEE Transactions on Cybernetics 46(8), 17491759 (2016)

36. L. Qi et al., an exception handling approach for privacy-preserving service recommendation failure in a cloud environment. Sensors 18, 7 (2018)

37. L. Qi, R. Wang, S. Li, Q. He, X. Xu, H. Chunhua, Time-aware distributed service recommendation with privacy-preservation. Inform Sci 480, 354-364 (2019)

38. Y Yu, L Qi, W Dou, J Yu. Privacy-preserving and scalable service recommendation based on SimHash in a distributed cloud environment. Complexity, Volume 2017, Article ID 3437854, 9 pages, 2017.

39. W Li, X Liu, J Liu, P Chen, S Wan, X Cui. On improving the accuracy with auto-encoder on conjunctivitis. Applied Soft Computing, 81, id: 105489, 2019.

40. M. Sekiguchi, K. Yano, T. Miyasaka, M. Ariyoshi, T. Kumagai, QoE-oriented WLAN: smart access control scheme for maximizing users' QoE satisfaction (2015 IEEE Wireless Communications and Networking Conference Workshops (WCNCW), New Orleans, 2015)

41. S. Wan, Y. Zhao, T. Wang, Z. Gu, Q.H. Abbasi, K.K.R. Choo, Multi-dimensional data indexing and range query processing via Voronoi diagram for internet of things. Future Generation Computer Systems 91, 382-391 (2019)

42. Wen Yuzhen, Yin Chuancun. On a dual model with barrier strategy, J Appl Mathematics, Volume 2012, 2012, Article ID 343794, 13 pages.

43. W Gong, L Qi, Y Xu. Privacy-aware multidimensional mobile service quality prediction and recommendation in distributed fog environment. Wireless Communications and Mobile Computing, vol. 2018, Article ID 3075849, 3075848 pages, 2018.

44. L. Qi, J. Yu, Z. Zhou, An invocation cost optimization method for web services in cloud environment. Scientific Programming, 2017, (2017-5-9) 2017 11, 1-9 (2017)

45. T. Saaty, Decision making with the analytic hierarchy process. Int.J.Servi.Sci 1(1), 83-98 (2008)

46. Xinsheng Du and Zengqin Zhao, On fixed point theorems of mixed monotone operators, fixed point theory and applications, Vol. 2011, ArticlelD 563136, 1-8 pages,doi:https://doi.org/10.1155/563136 (2011.02)

47. L. Qi, W. Dou, J. Chen, Weighted Principal component analysis-based service selection method for multimedia services in cloud. Computing 98(1), 195$214(2016)$

48. Panabit, http://www.panabit.com. Accessed May 2019.

49. Hoffman, Steve, Apache flume: distributed log collection for Hadoop, Packt Publishing Ltd, 2013.
50. L. Qi, Y. Chen, Y. Yuan, S. Fu, X. Zhang, X. Xu, A QoS-aware virtual machine scheduling method for energy conservation in cloud-based cyber-physical systems. W W W J (2019)

51. Vohra, Deepak, Using Apache Sqoop, Pro Docker. Apress, 2016.

52. G. Wenbo, Z. Xingying, Research on embedded data acquisition and processing based on ARM. J Computational Theoretical Nanosci 13(10), 6764-6768 (2016)

\section{Publisher's Note}

Springer Nature remains neutral with regard to jurisdictional claims in published maps and institutional affiliations.

\section{Submit your manuscript to a SpringerOpen ${ }^{\circ}$ journal and benefit from:}

- Convenient online submission

- Rigorous peer review

- Open access: articles freely available online

High visibility within the field

- Retaining the copyright to your article

Submit your next manuscript at $\boldsymbol{\nabla}$ springeropen.com 